\title{
CD30 Expression in Germinal Center B-cell-like and non-Germinal Center B-cell-like Subtypes of Diffuse Large B-cell Lymphoma
}

\author{
Patricia Fransisca Julianty ${ }^{1}$, Maria Francisca Ham ${ }^{1,2}$, Kusmardi Kusmardi ${ }^{1 *}$, Agnes Stephanie Harahap ${ }^{1,2}$ \\ ${ }^{1}$ Department of Anatomical Pathology, Faculty of Medicine, Universitas Indonesia, Indonesia; ${ }^{2}$ Department of Anatomical \\ Pathology, Cipto Mangunkusumo National Central General Hospital, Indonesia
}

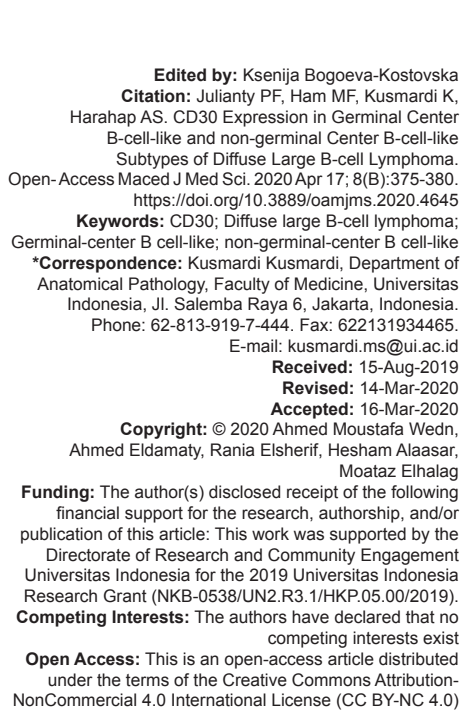

\begin{abstract}
BACKGROUND: Diffuse large B-cell lymphoma (DLBCL) is the most common and most heterogeneous type of nonHodgkin lymphoma. With current therapeutic modalities, $30 \%-40 \%$ of DLBCL cases still experience recurrence. The discovery of CD30 expression in DLBCL in several studies has opened up alternative opportunities for new targe
\end{abstract} therapies.

AIM: This cross-sectional study aimed to determine CD30 expression in DLBCL and its difference in expression in germinal center B-cell such as (GCB) and non-GCB subtypes.

METHODS: The sample consisted of 25 GCB and 25 non-GCB cases based on immunohistochemical examination performed at Cipto Mangunkusumo Hospital from 2014 to 2017. CD30 staining was carried out and assessed using tumor cells percentage with positive cutoff values of $>0 \%,>10 \%$, and $>20 \%$.

RESULTS: Positive CD30 expression was obtained in $8(16 \%), 4(8 \%)$, and $3(6 \%)$, out of 50 DLBCL cases with positive cutoff values of $>0 \%,>10 \%$, and $>20 \%$, respectively. We performed Fisher's exact test to determine CD30 expression in the GCB and non-GCB subtypes and found no significant difference with $p>0.05$.

CONCLUSION: Our study found no significant difference between CD30 expression in the GCB and non-GCB subtypes of DLBCL.

\section{Introduction}

Lymphoma is a primary malignancy in the lymph nodes and lymphoid tissue. Based on its histological type, lymphoma is divided into two major groups, non-Hodgkin Lymphoma (NHL) and $\mathrm{HL}$ [1]. NHL can originate from $\mathrm{B}$ lymphocytes, T lymphocytes, and NK cells [2]. According to the WHO Classification of Tumours of Haematopoietic and Lymphoid Tissues, there are 57 entities categorized as $\mathrm{NHL}$, and the most common is diffuse large B-cell lymphoma (DLBCL) [3]. The prevalence of DLBCL is $25-30 \%$ in developed countries and even higher in developing countries (42.5\%) [3], [4]. Data from the Division of Hematolymphoid and Bone Marrow, Department of Anatomical Pathology, Faculty of Medicine Universitas Indonesia - Cipto Mangunkusumo National General Hospital recorded DLBCL as the most common type of lymphoma (66.34\%) of all lymphomas.

DLBCL is the most heterogeneous type of NHL. According to the WHO Classification of Tumors of
Hematopoietic and Lymphoid Tissues, it is categorized into different morphological variants, molecular subtypes, and distinct disease entities [3]. Based on gene-expression profiling (GEP), DLBCL is classified into germinal-center $B$ cell-like (GCB), activated B-cell $(A B C)$, and type 3 subtypes. The GCB subtype has better survival than $A B C$. The type 3 subtype is very heterogeneous and cannot be classified into existing groups but has a poor prognosis such as ABC. GEP is not practical for daily use, so immunohistochemistry (IHC) is used with a substitute algorithm to classify GCB and non-GCB subtypes [5].

DLBCL is more common in older people with a median age in the seventh decade and occurs more often in men. It is generally accompanied by rapid enlargement of lymph nodes or extranodal masses [3]. These lymphomas have an aggressive nature with a survival of $<1$ year in patients who do not receive therapy [6]. The addition of rituximab to standard cyclophosphamide, adriamycin, vincristine, and prednisone (CHOP) therapy improves the overall 
survival (OS) of DLBCL patients. However, one-third of patients still experience recurrence after therapy [7].

Even though DLBCL has been classified, the groups are still heterogeneous. For example, in the GCB subtype, which generally has a good prognosis, MYC/BCL2 double-hit B cell lymphoma can be found, which possesses a very aggressive clinical temperament [7], [8]. Many studies have been carried out to obtain other indicators/ biomarkers that can help classify DLBCL more specifically to provide prognostic clues and open up opportunities for new therapeutic approaches [7].

The discovery of CD30 expression in DLBCL in several studies has opened alternative opportunities for new therapeutic targets. CD30 is a member of tumor necrosis factor receptor superfamily, originally known as the cell surface marker of Reed-Sternberg and Hodgkin cells in classic $\mathrm{HL}(\mathrm{CHL})$. These receptors are also expressed by several $T$ cells and $B$ cells in NHL such as anaplastic large cell lymphoma (ALCL), primary cutaneous CD30+ T-cell lymphoma, lymphoproliferative disorders, and primary mediastinal large B-cell lymphoma [7], [9]. CD30 plays a role in increasing and maintaining memory $\mathrm{T}$ and $\mathrm{B}$ cells, $B$ cell proliferation, and increasing immunoglobulin production [7]. CD30 expression in DLBCL has been investigated by several researchers with varying results (10-27\%) depending on the positive cutoff value used $(>0-20 \%$ ) [7], [10], [11], [12], [13]. Research by Xu et al. and Wang et al. used more than one cutoff value and obtained different statistical test results with the use of different cutoff values [11], [13]. CD30 expression can be one of the targets for immunotherapy specific to cancer cells. Anti-CD30 monoclonal antibody-drug conjugate, brentuximab vedotin, has now been approved for the use in ALCL and CHL patients with recurrence [7], [14].

To the best of our knowledge, this is the first Indonesian study to analyze CD30 expression in DLBCL. From the results of the previous studies, the prevalence of CD30 expression in DLBCL and its differences between the GCB and non-GCB subtypes was still varied, as was the positive cutoff values used. The role of CD30 expression in DLBCL is also still unknown. We hope that further understanding of CD30 expression in DLBCL, with specifications in each subtype, will improve knowledge about the biological behavior of DLBCL and help determine adjustments in therapeutic approaches.

\section{Methods}

\section{Patients}

The target population of the study was the DLBCL. The attainable population was all DLBCL cases that had gone through immunohistochemical examination in Cipto Mangunkusumo National General Hospital from January 2014 to December 2017. The selection of samples was done by a consecutive sampling of cases that met the inclusion and exclusion criteria. The inclusion criteria were DLBCL cases on which IHC staining of CD20, CD10, Bcl-6, and MUM1 had been performed for determination of GCB and non-GCB subtypes. Exclusion criteria were cases with paraffin blocks that were not found or inadequate and DLBCL cases originating from the mediastinum or accompanied by mediastinal masses. A sample of 50 DLBCL cases was obtained, consist of 25 GCB and 25 non-GCB cases. Approval for the study was granted by the Ethics Committee of the Faculty of Medicine, University of Indonesia (0960/UN2.F1/ETIK/2018).

\section{IHC staining of CD30}

CD30 staining was done using a concentration ratio of 1:100. Preparations were heated on a slide warmer for $30 \mathrm{~min}$ at $60^{\circ} \mathrm{C}$. The preparations were then deparaffinized with xylol $(3 \times 3 \mathrm{~min})$ followed by rehydration with decreasing alcohol concentrations (ethanol, 96\% alcohol, and 70\% alcohol, 3 min each) and washed with running water for 2 min. Antigen retrieval procedure was done using a Tris EDTA buffer, $\mathrm{pH} 9.0$, within a decloaking chamber at $96^{\circ} \mathrm{C}$ for $10 \mathrm{~min}$, then cooled and washed with phosphate buffered saline (PBS) at $\mathrm{pH} 7.4$ for 2 min. The tissue was then marked with a Pap-pen. Endogenous peroxidase blocking was carried out for $10 \mathrm{~min}$, followed by a 2-time washing with $\mathrm{pH}$ 7.4 PBS. Background blocking was performed for $10 \mathrm{~min}$ and washed with $\mathrm{pH} 7.4$ PBS. Preparations were then incubated with CD30 primary antibodies (Ki-1 Antigen); Dako clone Ber-H2 code M0751, for 30 min and washed with pH 7.4 PBS 4 times, continued by incubation with Novolink polymer secondary antibodies for $30 \mathrm{~min}$ and washed with $\mathrm{pH}$ 7.4 PBS 3 times. Preparations were incubated with a mixture of $1 \mathrm{ml}$ diaminobenzidine tetrahydrochloride (DAB) substrate (high contrast) and 1 drop $(50 \mu \mathrm{l})$ of DAB chromogen for $30 \mathrm{~s}$ and washed with running water for 5 min. Counterstain with Mayer hematoxylin for $1 \mathrm{~min}$ was performed, and the preparation was washed with running water for 5 min. Bluing was carried out using lithium carbonate for $10 \mathrm{~s}$ and washed with running water for 5 min. Dehydration with increasing alcohol concentrations (70\% alcohol, 96\% alcohol, and ethanol) followed. The clearing was done using xylol I, II, and III, 3 min each. Negative and positive controls were included every time staining was performed. The positive control for CD30 came from an ALCL case.

\section{Assessment of CD30 expression in staining}

CD30 expression will show as staining on the membrane and/or paranuclear Golgi dots [3], [11]. 
The assessment was carried out by identifying tumor areas that were well-stained, followed by taking several photographs in the areas where most stains were found. Preparations were assessed with $\times 400$ using a Leica ICC50 HD microscope until 500 tumor cells were obtained. Photographs were then processed using Image $\mathrm{J}$, and the identified cells were calculated using a cell counter. We counted both tumor cells that expressed CD30 and those that did not. CD30 expression was assessed in percentage and was categorized as positive if it fell above the cutoff values of $>0 \%,>10 \%$, or $>20 \%$.

\section{Statistical analysis}

Data processing was done using SPSS version 20.0. Statistical analysis used a comparative hypothesis test to determine differences in CD30 expression between GCB and non-GCB DLBCL subtypes using Fisher's exact test. $p<0.05$ was considered statistically significant.

\section{Results}

\section{Demographic characteristics}

Out of 50 subjects, DLBCL was found to occur more often at the age of $\leq 60$ years $(64 \%)$ with an age range of 13-83 years, with a median age of 56 years and a man-to-woman ratio of 1.2:1. The most common tumor sites are extranodal areas (86\%) such as the tonsils, eyes, nasopharynx, and the sinonasal area (Table 1).

Table 1: Demographic characteristics of study subjects

\begin{tabular}{cllll}
\hline Variable & $\begin{array}{l}\text { GCB subtype } \\
(\mathrm{n}=25)\end{array}$ & $\begin{array}{l}\text { Non-GCB subtype } \\
(\mathrm{n}=25)\end{array}$ & Total $(\mathrm{n}=50)$ & Percentage \\
\hline Age & & & & \\
$\quad \leq 60$ & 20 & 12 & 32 & 64 \\
$\quad>60$ & 5 & 13 & 18 & 36 \\
Sex & & 9 & 27 & 54 \\
$\quad$ Male & 18 & 16 & 23 & 46 \\
$\quad$ Female & 7 & & 7 & 14 \\
Tumor site & & 2 & 43 & 86 \\
$\quad$ Nodal & 5 & 23 & & \\
$\quad$ Extranodal & 20 & &
\end{tabular}

The most common morphological variant found in the sample was the centroblastic variant $(92 \%)$. The immunoblastic variant was found in three cases (6\%), and one case was found with an anaplastic variant (Figure 1).

\section{CD30 expression}

CD30 expression was assessed as the percentage of tumor cells that were stained positive on the membrane and/or perinuclear dots. The positive control was obtained from an ALCL case (Figure 2).

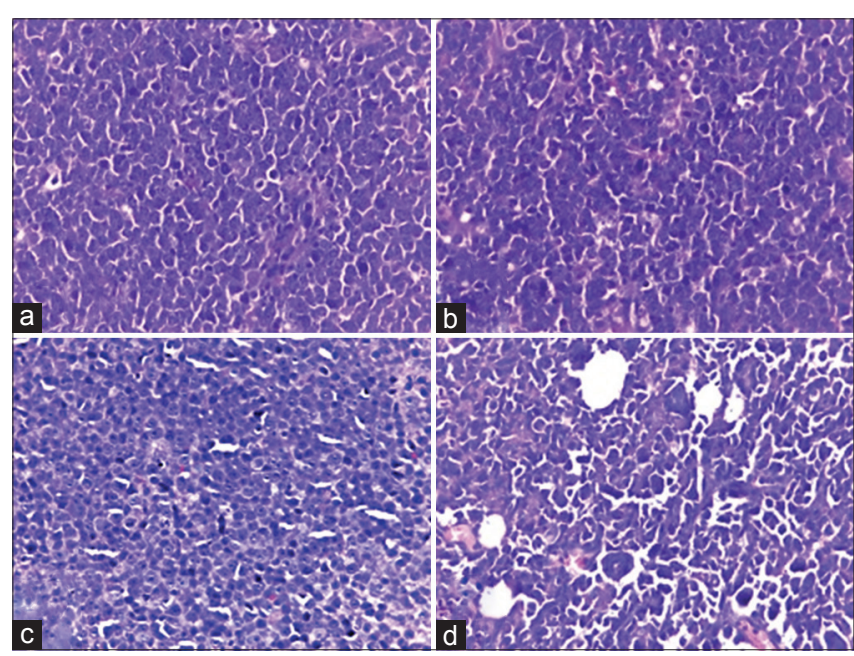

Figure 1: Morphological variants of diffuse large B-cell lymphoma. (a and b) Centroblastic variant, (c) immunoblastic variant, (d) anaplastic variant $(\times 400)$

Weak, moderate, and strong positive stains were categorized as positive. The cutoff values used to determine positivity were $>0 \%,>10 \%$, and $>20 \%$ of tumor cells showing positive CD30 staining, according to existing literature [7], [10], [11], [12], [13].

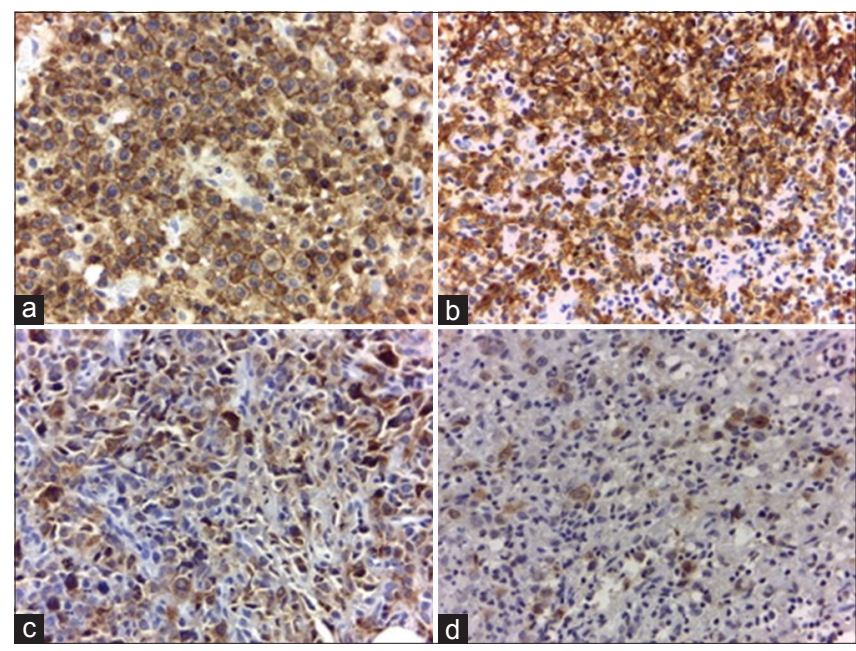

Figure 2: Positive control of $C D 30$ staining and positive $C D 30$ expression using a cutoff value of $>20 \%$. (a) Positive control using anaplastic large cell lymphoma, (b) non-germinal center B-cell like $(G C B)$ diffuse large $B$-cell lymphoma (DLBCL) with a positive value of $97.4 \%$, (c) non-GCB DLBCL with a positive value of $62.6 \%$ (d) GCB $D L B C L$ with a positive value of $29.4 \%(\times 400)$

The use of the $>20 \%$ cutoff value obtained positive CD30 expression in three (6\%) out of 50 cases, classified into two non-GCB cases, and one GCB case. Morphological variation showed two cases with a centroblastic variant and one case with an anaplastic variant (Figure 2).

The use of the $>10 \%$ cutoff value resulted in the presence of positive CD30 expression in 4 (8\%) out of 50 cases, classified into three non-GCB cases, and one GCB case. Two cases had a centroblastic variant, one case an anaplastic variant, and another case an immunoblastic variant (Figure 3) 


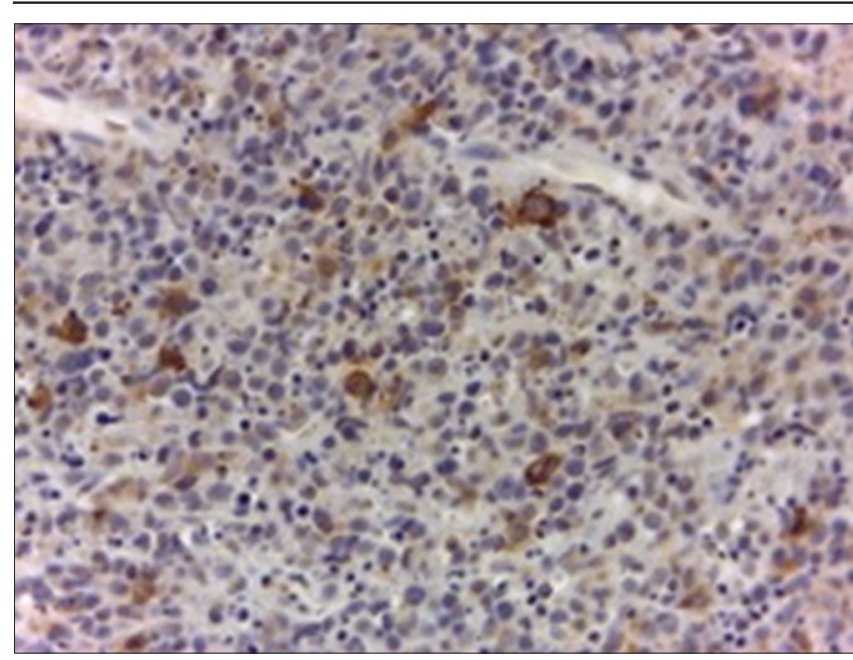

Figure 3: Positive CD30 expression using a cutoff value of $>10 \%$. Non-germinal center B-cell like diffuse large B-cell lymphoma with a positive value of $16 \%(\times 400)$

A cutoff value of $>0 \%$ yielded positive CD30 expression in $8(16 \%)$ out of 50 cases, classified into five non-GCB cases, and three GCB cases. The morphological variation included six cases with a centroblastic variant, one case with an anaplastic variant, and one case with an immunoblastic variant (Figure 4).

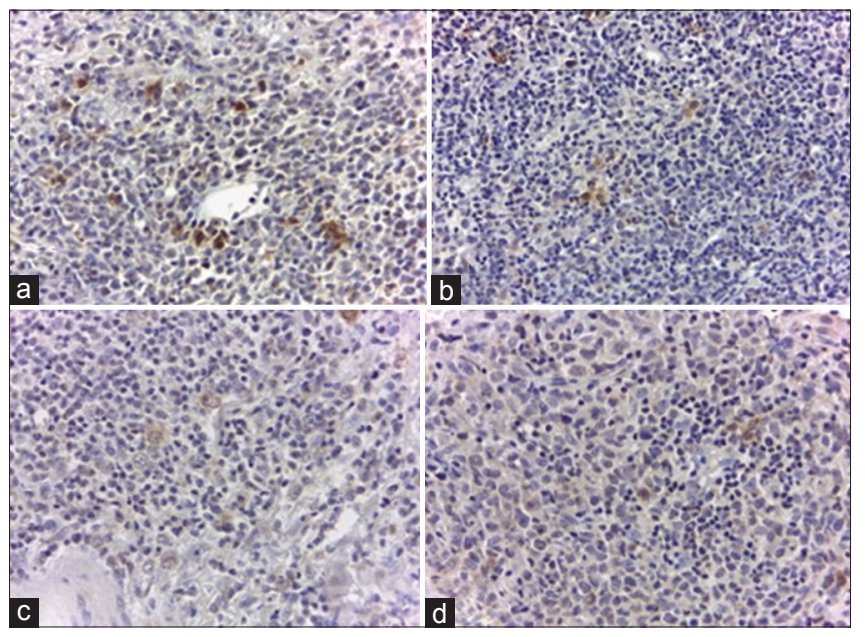

Figure 4: Positive $C D 30$ expression using a cutoff value of $>0 \%$. (a) Non-germinal center B-cell like (GCB) diffuse large B-cell lymphoma (DLBCL) with a positive value of $6.6 \%$, (b) GCB DLBCL with a positive value of $6 \%$, (c) GCB DLBCL with a positive value of $3.4 \%$, (d) non-GCB DLBCL with a positive value of $3 \%(\times 400)$

Fisher's exact test revealed no significant difference between CD30 expression in GCB DLBCL and non-GCB DLBCL, with $p>0.05$ using all cutoff values. Statistical tests were also conducted to see differences in CD30 expression in GCB and non-GCB subtypes when associated with age, sex, tumor site, and results of IHC staining (CD10, Bcl-6, and MUM1). Significant differences in CD30 expression were found in sex variables using the $>10 \%$ cutoff value. There were no significant differences in CD30 expression when associated with age, tumor site, and IHC staining results (CD10, Bcl-6, and MUM1) using all cutoff values (Table 2).

\section{Discussion}

DLBCL occurs in $25-35 \%$ of all adult NHLs in developed countries and $42.5 \%$ in developing countries [3], [4]. Its incidence increases by $3 \%-4 \%$ every year [15]. DLBCL is commonly found in old age with a median age in the seventh decade, although it can also have its onset in childhood and early adulthood [3], [16]. This study involved subjects with an age range of 13-83 years with a median of 56 years. The median age is younger compared to several studies in Western countries with a median age of 60-64 years, but quite similar with studies by Hao et al. and Gong et al. in China with a median age of 49 and 59 years [7], [11], [12], [13]. Our study found that DLBCL was more prevalent at $\leq 60$ years $(64 \%)$, as similarly found by Chen et al. ( $75 \%$ at $<60$ years of age) [17].

Our study also found a higher DLBCL incidence in men with a ratio of 1.2:1 compared to women. This is in accordance with the existing literature, which expressed similar ratios ranging from $1.4: 1$ to $1.9: 1$ [7], [11], [12], [18]. Forty percent of DLBCL cases are located in extranodal sites [3], while this study found that $86 \%$ of its DLBCL cases were located in extranodal sites. This could be due to the lack of clinical data that rendered it difficult to know nodal involvement in this study.

The addition of rituximab to $\mathrm{CHOP}$ standard therapy has improved the OS of DLBCL patients; however, one-third of DLBCL patients still experience recurrence or resistance after therapy [7]. CD30 expression found in DLBCL has opened opportunities for new targeted therapy approaches. CD30 expression in DLBCL in several studies showed varied results $(10-27 \%)$ depending on the positive cutoff value used $(>0-20 \%)$ [7], [10], [11], [12], [13]. In a study by Xu et al., two cutoff values were used $(>0 \%$ and $>20 \%)$, and different statistical test results were obtained between the two cutoff values [13]. Wang et al. used three different cutoff values $(>0 \%,>20 \%$, and $>40 \%)$ and also obtained different statistical test results. Therefore, in this study, three cutoff values were used. The cutoff values used were taken from existing literature [7], [10], [11], [12], [13].

This study assessed CD30 expression using IHC staining. A positive result is indicated by tumor cell membranes and/or paranuclear Golgi dots staining [3], [11]. The results showed that DLBCL expressed CD30 in 16\%, $8 \%$, and $6 \%$ of the 50 cases examined using positive cutoff values of $>0 \%,>10 \%$, and $>20 \%$, respectively. Previous research using a cutoff value of $>20 \%$ received CD30 expression results of $12-22 \%$ [13], [18]. Salas et al. used a cutoff value of 
Table 2: Association between CD30 expression and DLBCL subtype, demographic characteristics, and immunohistochemical staining (CD10, Bcl-6, and MUM1)

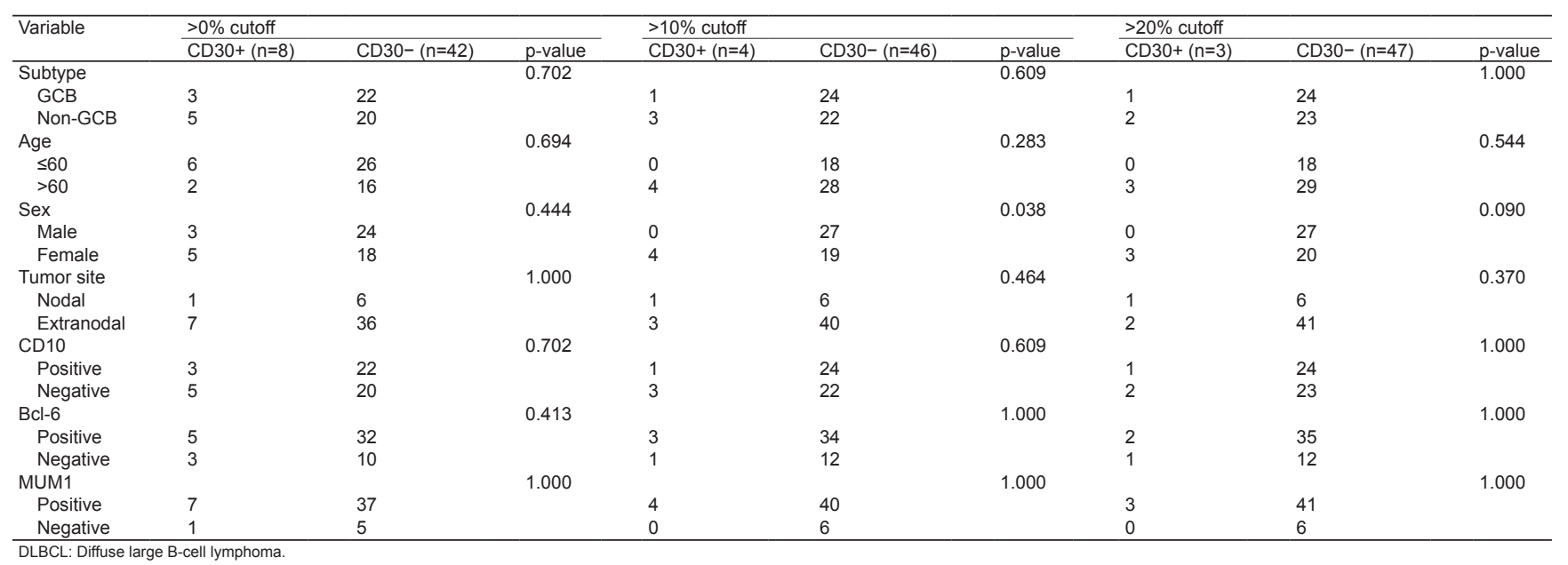

$>10 \%$ and obtained a $27 \%$ result [10]. The use of a $>0 \%$ cutoff value obtained results of 10-41\% [11], [13]. These varying results might be due to several confounding variables such as age, sex, and therapy. In a study that found positive CD30 expression in $41 \%$ of its subjects, primary antibodies with a different clone were used (JCM182 Leica) [18].

Fisher's exact test was performed to assess the difference between CD30 expression in GCB and non-GCB subtypes using positive cutoff values of $>0 \%,>10 \%$, and $>20 \%$. All cutoff values yielded no statistically significant difference in the proportion of CD30 expression. Wang et al. and Zuluaga et al. found that CD30 was more likely to be expressed in the non-GCB subtype, although no statistical significance was found [18], [19]. A study by Salas et al. found a significant difference in the proportion of CD30 expression between GCB and non-GCB subtypes, with predominance in non-GCB subtypes [10]. Their study used a larger sample size. Hao et al. also found a significant difference in the proportion of CD30 expression between the two subtypes with the predominance of non-GCB subtypes. Their study used primary antibodies (clone: EP154) [12]. These varying results were likely to be caused by differences in sample size, antibody clones, and heterogeneity of patients.

Zuluaga et al. found that in cases with positive BCL2 staining, CD30 expression was found 3.9 times higher in the non-GCB subtype than in the GCB subtype. They also found that the frequency of positive CD30 expression in DLBCL with positive BCL2 staining was higher than the overall CD30 expression frequency in the study. BCL2 seems to play a role in CD30 expression, where CD30 is expressed in a greater proportion in the non-GCB subtype and younger ages if BCL2 is also expressed. This interaction is associated with the NF-kB transcription factor pathway [19]. Gong et al. obtained a positive relationship between CD30 expression and BCL2 rearrangement and a negative relationship with BCL2 protein expression in the non-GCB subtype. In their study, CD30 expression and MYC rearrangement were also found to be mutually exclusive [11]. Studies conducted by Zaluaga et al. and Gong et al. showed that the role of CD30 expression in DLBCL was also influenced by several other things. Therefore, further research is needed to understand the link between CD30 expression in DLBCL and expressions of BCL2 and MYC.

The proportion of CD30 expression is not significantly different when associated with age and tumor site using all three cutoff values. The studies by Wang et al. and Hao et al. also did not obtain any significant difference when associated with age [12], [18]. In this study, there was a difference in the proportion of CD30 expression when associated with $\operatorname{sex}(p=0.038)$. Using a cutoff value of $>10 \%$, CD30 expression was found to be more pronounced in females. The results were however not significant when using $>0 \%$ and $>20 \%$ cutoff values $(p=0.444$ and $p=0.090)$. CD30 expression was not significantly different between IHC staining results of CD10, Bcl-6, and MUM1.

Gong et al. found that CD30 expression was more commonly found in cases with centroblastic and anaplastic variants [11]. Similarly, we found that the largest number of CD30-positive preparations was of the centroblastic variant. In one case with an anaplastic variant, CD30 expression was also obtained.

Based on our results, it was found that $16 \%$ of DLBCL cases had the potential to get anti-CD30 target therapy. The antibody-drug conjugate brentuximab vedotin (SGN-35) which combines monoclonal anti-CD30 antibodies with antitubulin agent monomethyl auristatin $E$ can be a therapeutic choice for CD30positive DLBCL cases. This therapeutic modality is very promising due to the limited expression of CD30 in only some $\mathrm{T}$ cells and B cells in the parafollicular area in healthy individuals, making it more selective [19]. Brentuximab vedotin has gone through a second-phase clinical trial for CD30-positive DLBCL patients who experienced recurrence, and results have shown that $40 \%$ of patients achieved a clinical response and $17 \%$ 
achieved full remission. However, it was said that no correlation was found between the average successes of therapeutic responses with CD30 expression levels, even though all of the included patients had CD30 expression [20]. These results indicate that the use of a cutoff value of $>0 \%$ in daily clinical practice can ensure that more patients benefit from the targeted therapy.

\section{Conclusion}

GCB and non-GCB subtypes of DLBCL did not have any significant difference in CD30 expression.

\section{References}

1. Rinaldi I, Hardjolukito ES, Prajogi GB, Giselvania A, Nuhonni SA Panduan Penatalaksanaan Limfoma non Hodgkin. Jakarta: Komite Nasional Penanggulangan Kanker; 2015. p. 1-8.

2. Chiu BC, Hou N. Epidemiology and etiology of nonHodgkin lymphoma. In: Evens AM, Blum KA, editors. NonHodgkin Lymphoma Pathology, Imaging, and Current Therapy. Switzerland: Springer; 2015. p. 1-25. https://doi. org/10.1007/978-3-319-13150-4_1

3. Gascoyne RD, Campo E, Jaffe ES, Staudt LM. Diffuse large B-cell lymphoma, NOS. In: Swerdlow SH, Campo E, Harris NL, Jaffe ES, Pileri SA., editors. WHO Classification of Tumours of Hematopoietic and Lymphoid Tissues. 4th ed. London: IARC; 2017. p. 291-7.

4. Perry AM, Diebold J, Nathwani BN, MacLennan KA, MüllerHermelink HK, Bast $M$, et al. Non-Hodgkin lymphoma in the developing world: Review of 4539 cases from the International Non-Hodgkin Lymphoma Classification Project. Haematologica. 2016;101(10):1244-50. https://doi.org/10.3324/ haematol.2016.148809

PMid:27354024

5. Hans CP, Weisenburger DD, Greiner TC, Gascoyne RD, Delabie J, Ott G, et al. Confirmation of the molecular classification of diffuse large B-cell lymphoma by immunohistochemistry using a tissue microarray. Blood. 2004;103(1):275-82. https:// doi.org/10.1182/blood-2003-05-1545

PMid:14504078

6. Rovira J, Valera A, Colomo L, Setoain X, Rodríguez S, MartínezTrillos A, et al. Prognosis of patients with diffuse large B cell lymphoma not reaching complete response or relapsing after frontline chemotherapy or immunochemotherapy. Ann Hematol. 2015;94(5):803-12. https://doi.org/10.1007/s00277-014-2271-1 PMid:25501975

7. Hu S, Xu-Monette ZY, Balasubramanyam A, Manyam GC Visco C, Tzankov A, et al. CD30 expression defines a novel subgroup of diffuse large B-cell lymphoma with favorable prognosis and distinct gene expression signature: A report from the International DLBCL Rituximab-CHOP Consortium Program Study. Blood. 2013;121(14):2715-24. https://doi.org/10.341 O/f.717989810.793472956

PMid:23343832

8. Aukema SM, Siebert R, Schuuring E, van Imhoff GW, KluinNelemans HC, Boerma EJ, et al. Double-hit B-cell lymphomas. Blood. 2011;117(8):2319-31. https://doi.org/10.1182/ blood-2010-09-297879

PMid:21119107

9. de Leval L, Gaulard P. CD30+ Iymphoproliferative disorders. Haematologica. 2010;95(10):1627-30. https://doi.org/10.3324/ haematol.2010.029256

PMid:20884717

10. Salas Q, Climent F, Tapia G, Riasol M, Mercadal S, Domingo E. CD30 expression in diffuse large B-cell lymphoma correlates with non-GCB subtype but does not have prognostic impact in patients treated with first line R-CHOP/R-CHOP-like. Hematol Oncol. 2017;35:290-1. https://doi.org/10.1002/hon.2439_19

11. Gong QX, Wang Z, Liu C, Li X, Lu TX, Liang JH, et al. CD30 expression and its correlation with MYC and BCL2 in de novo diffuse large B-cell lymphoma. J Clin Pathol. 2018;71(9):795801. https://doi.org/10.1136/jclinpath-2018-205039 PMid:29666157

12. Hao $\mathrm{X}$, Wei $\mathrm{X}$, Huang $\mathrm{F}$, Wei $\mathrm{Y}$, Zeng $\mathrm{H}, \mathrm{Xu} \mathrm{L}$, et al. The expression of CD30 based on immunohistochemistry predicts inferior outcome in patients with diffuse large B-cell lymphoma. PLoS One. 2015;10(5):e0126615. https://doi.org/10.1371/ journal.pone. 0126615

PMid:25974110

13. Xu J, Oki Y, Saksena A, Desai P, Lin P, Tang G, et al. CD30 expression and prognostic significance in $\mathrm{R}-\mathrm{EPOCH}$-treated patients with diffuse large B-cell lymphoma. Hum Pathol. 2017;60:160-6. https://doi.org/10.1016/j.humpath.2016.10.009 PMid:27816715

14. van der Weyden CA, Pileri SA, Feldman AL, Whisstock J, Prince $\mathrm{HM}$. Understanding CD30 biology and therapeutic targeting: A historical perspective providing insight into future directions. Blood Cancer J. 2017;7(9):e603. https://doi.org/10.1038/bcj.2017.85 PMid:28885612

15. Gouveia GR, Siqueira SA, Pereira J. Pathophysiology and molecular aspects of diffuse large B-cell lymphoma. Rev Bras Hematol Hemoter. 2012;34(6):447-51. https://doi. org/10.5581/1516-8484.20120111

PMid:23323070

16. Martelli M, Ferreri AJ, Agostinelli C, Di Rocco A Pfreundschuh M, Pileri SA. Diffuse large B-cell lymphoma. Crit Rev Oncol Hematol. 2013;87(2):146-71. https://doi. org/10.1016/j.critrevonc.2012.12.009 PMid:23375551

17. Chen $P$, Jiang $M Q$, Ruan $X Z$, Cai NL, Ye XW, Pan $Y N$, et al The phenotypes of germinal center B-cell-like (GCB) and non-GCB failed to predict the survival of patients with diffuse large B-cell lymphoma in the rituximab era. Int J Clin Exp Med. 2017:10:13564-70.

18. Wang XJ, Seegmiller AC, Reddy NM, Li S. CD30 expression and its correlation with MYC rearrangement in de novo diffuse large B-cell lymphoma. Eur J Haematol. 2016;97(1):39-47. https://doi.org/10.1111/ejh.12680

PMid:26340843

19. Campuzano-Zuluaga G, Cioffi-Lavina M, Lossos IS, ChapmanFredricks JR. Frequency and extent of CD30 expression in diffuse large B-cell lymphoma and its relation to clinical and biologic factors: A retrospective study of 167 cases. Leuk Lymphoma. 2013;54(11):2405-11. https://doi.org/10.3109/1042 8194.2013.778407 PMid:23432725

20. Bartlett NL, Sharman JP, Oki Y, Advani RH, Bello CM Winter JN, et al. A phase 2 study of brentuximab vedotin in patients with relapsed or refractory CD30-positive non-Hodgkin lymphomas: interim result in patient with DLBCL and other B-Cell lymphoma. Blood. 2013;122:848. https://doi.org/10.1182/ blood.v122.21.848.848 